\section{Hacia una metología integral para el desarrollo de la literacidad en la educación intercultural bilingüe}

Towards a

Comprehensive

Methodology for

the Development

of Literacy in

Intercultural Bilingual

Education
Rumo a uma metodologia

compreensiva para 0

desenvolvimento do

letramento na educação

intercultural bilíngue

Primer semestre de $2022 \cdot$ pp. 185-198

Segunda época

N.

María Cristina Castro Azuara* https://orcid.org/0000-0002-0887-3143

Laura Aurora Hernández Ramírez** https://orcid.org/0000-0003-4092-5625

Refugio Nava Nava*** https://orcid.org/0000-0002-5721-0426
Para citar este artículo

Castro-Azuara, M. C., Hernández-Ramírez, L. A. y Nava-Nava, R. (2022). Hacia una metodología integral para el desarrollo de la literacidad en la educación intercultural bilingüe. Folios, (55). https://doi.org/10.17227/folios.55-12958
Doctora en Ciencias del Lenguaje. Universidad Autónoma de Tlaxcala, México.

Correo: mariacristina.castro@uatx.mx

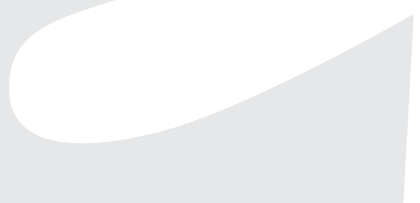

Doctora en Ciencias del Lenguaje. Universidad Autónoma de Tlaxcala, México.

Correo: lauraaurora.hernandez@uatx.mx

*** Doctor en Antropología. Universidad Autónoma de Tlaxcala, México.

Correo: refugio.nava@uatx.mx
Artículo recibido

$09 \cdot 12 \cdot 2020$

Artículo aprobado $02 \cdot 07 \cdot 2021$ 


\title{
Resumen
}

En este artículo de reflexión se discute la pertinencia de establecer una metodología integral para la enseñanza de la lengua indígena en el contexto de la educación intercultural bilingüe en México. Partimos del planteamiento de que, si bien existen lineamientos para guiar el funcionamiento de la educación intercultural bilingüe, estos no se han concretado en metodologías para la enseñanza de la oralidad, lectura y la escritura de las lenguas originarias. Por lo mismo, a partir de una teoría fundamentada en los nuevos estudios de la literacidad y la pedagogía del género discursivo, proponemos abordar la enseñanza de las lenguas indígenas desde un enfoque sociosemiótico e intercultural basado en el género discursivo, pues, además de facilitar la adquisición de la lengua, permite a los estudiantes reconocerse como miembros activos en sus entornos inmediatos al reafirmar las prácticas de literacidad propias de su comunidad e integrarlas a las prácticas escolares. Concluimos que solo a través de un bilingüismo aditivo, guiado por metodologías y planes de intervención pedagógica fundamentados en una mirada social del lenguaje, se logrará que los niños y niñas indígenas ejerzan su derecho a participar de la lectura y escritura en sus propias lenguas y, simultáneamente, se involucren en prácticas sociales que les ayuden a construir conocimiento para acceder a mejores oportunidades de vida dentro y fuera de sus comunidades.

\section{Palabras clave}

educación multicultural bilingüe, enseñanza de la lengua indígena, literacidad, prácticas sociales, género discursivo.

\begin{abstract}
This reflection paper discusses the relevance of generating a comprehensive methodology for the teaching of indigenous languages in the context of intercultural bilingual education in Mexico. We start from the proposition that, although there are guidelines to conduct the operation of intercultural bilingual education to guarantee quality in education with cultural relevance for indigenous people, these have not been materialized ineffective methodologies for the teaching of orality, reading, and writing in the original languages. For this reason, based on a theoretical foundation from the New Literacy Studies and Genre-Based Pedagogy, we show the relevance of approaching the teaching of indigenous languages from an intercultural perspective which, in addition to facilitating the acquisition of the code, allows students to recognize themselves as active members in their immediate surroundings by learning the literacy practices of their community and integrating them into the school practices. We conclude that only through additive bilingualism, guided by methodologies and pedagogical intervention plans based on a social view of language, it will be possible for indigenous boys and girls to exercise their right to participate in reading and writing in their languages while getting involved in social practices that would help them build knowledge to access better life opportunities inside and outside their communities.
\end{abstract}

Keywords

multicultural bilingual education; teaching indigenous languages; literacy; social practices; discourse genre

\section{Resumo}

Neste artigo de reflexão discute-se a relevância de estabelecer uma metodologia integral para o ensino da língua indígena no contexto da educação intercultural bilíngue no México. Consideramos que, mesmo havendo diretrizes para o funcionamento da educação intercultural bilíngue, estas não se concretizam em metodologias eficazes para o ensino da oralidade, leitura e escrita das línguas originárias. Por isto, partindo de uma teoria fundamentada nos novos Estudos do Letramento e na pedagogia baseada no gênero discursivo, mostramos a relevância do ensino das línguas indígenas a partir de uma perspectiva sociosemiótica e intercultural baseada no gênero discursivo, pois, além de facilitar a aquisição da língua, permite que os estudantes se reconheçam como membros ativos nos contextos imediatos reafirmando as práticas de letramento da própria comunidade, integrando-as às práticas escolares. Concluímos que só através de um bilinguismo aditivo, guiado por metodologias e planos de intervenção pedagógica fundamentados em uma visão social da linguagem, conseguirá-se que os meninos e as meninas indígenas exerçam 
o direito que lhes é constitutivo para participarem da leitura e da escrita em suas próprias línguas e, simultaneamente, envolverem-se em práticas sociais que lhes ajudem a construir conhecimento para alcançar melhores oportunidades de vida dentro e fora das suas próprias comunidades.

\section{Palavras chave}

educação multicultural; ensino da língua indígena; letramento; práticas sociais; gênero discursivo

\section{Introducción}

Es un hecho que las prácticas interculturales solo son posibles en contextos de igualdad social, económica, lingüística y cultural. Si bien la ley general de educación, promulgada en el 2019 en el marco de la Nueva Escuela Mexicana, establece en su artículo 56 los derechos educativos, culturales y lingüísticos a todas las personas, pueblos y comunidades indígenas $o$ afromexicanas, migrantes y jornaleros, la educación intercultural bilingüe continúa sin lograr su objetivo de promover una educación de calidad, incluyente y con pertinencia cultural para los pueblos indígenas $\mathrm{y}$ afrodescendientes, en contextos multiculturales y multilingües específicos.

No son pocas las voces que señalan que la incorporación oficial de las lenguas originarias a la educación pública mexicana ha implicado retos que no han sido abordados de manera sistemática y efectiva (Barriga, 2018; Dietz y Mateos, 2011; Feltes, 2017; Hamel et al., 2016 y Muñoz, 2017). También son muchos los aspectos que determinan el fracaso de la enseñanza de la lectura y la escritura en los contextos multilingües, pero, sin duda, uno de ellos es la ausencia de una metodología consensada e integral que sustente la enseñanza de las prácticas sociales discursivas en lenguas originarias y adquiridas y permita que los niños, jóvenes y docentes convivan en un verdadero bilingüismo equilibrado dentro de una real educación intercultural. Aunque la asignatura Lengua Indígena tiene propósitos y metas bien definidos orientados por el enfoque de prácticas sociales del lenguaje que se enarbola en el país, está aún lejos de cumplir sus metas debido a la ausencia de una orientación didáctica que permita a los profesores contar con materiales originales y situados que cumplan con la exigencia de apegarse a la cultura de los estudiantes en las distintas dimensiones de la educación formal a través de la enseñanza, el uso y la conservación de la lengua indígena como lengua materna o adquirida.

En este trabajo defendemos la idea de que la enseñanza de la lengua materna y adquirida en contextos multilingües es posible si se dejan de lado los discursos condescendientes que, lejos de ser inclusivos, fomentan un bilingüismo desequilibrado, en lugar de proyectar políticas lingüísticas orientadas al desarrollo de procesos de literacidad — que incluyen la oralidad- enfocados en el reconocimiento y la promoción de la diversidad, tal como lo expresan los objetivos la Nueva Escuela Mexicana (Diario Oficial de la Federación [DOF], 2019). Partimos del planteamiento de que el aprendizaje de la lengua está sustentado en las relaciones que se establecen entre el aprendizaje individual y las prácticas de las 
comunidades culturales, toda vez que los hablantes se involucran en aprendizajes situados en el marco de prácticas específicas que van cambiando a lo largo de sus vidas (Rogoff y Gutiérrez, 2010; Wenger, 2001). Asimismo, siguiendo los postulados de los nuevos estudios de literacidad (Cassany, 2008; Gee, 2006; Heller, 2007; Zavala, 2011), suponemos que la lengua es una práctica que forma parte de un conjunto más complejo de actividades sociales, en la cual los sujetos, como actores sociales, echan mano de recursos lingüísticos para lograr propósitos específicos bajo condiciones particulares; y que el aprendizaje de la lengua, en consecuencia, está estrechamente relacionado con aspectos socioculturales como la identidad, las relaciones sociales y el manejo de artefactos semióticos. Así, siguiendo este planteamiento, en la primera sección del artículo revisamos los métodos y enfoques de enseñanza que tradicionalmente se han seguido en el contexto de la educación intercultural bilingüe para, en el segundo apartado, detenernos en las posibilidades de un enfoque basado en los nuevos estudios de literacidad y la teoría pedagógica del género como marcos de referencia para la planeación, el desarrollo y la implementación de metodologías más eficaces para la enseñanza de la oralidad y la escritura. Posteriormente, delinearemos cuatro principios que, desde esta perspectiva, deben guiar la construcción de una malla curricular en el contexto de la educación intercultural bilingüe en nuestro país. Finalmente, en las conclusiones invitamos al lector a reflexionar sobre este complejo problema de política lingüística, de concepción discursiva del lenguaje y su implementación didáctica.

\section{Proyectos de enseñanza de la lengua en la educación intercultural bilingüe en México}

A pesar de que desde distintos espacios se han generado leyes y reglamentos sobre el papel de las lenguas indígenas en la educación intercultural bilingüe (DOF, 2019; Organización de las Naciones Unidas para la Educación, la Ciencia y la Cultura [Unesco], 2006; Secretaría de Educación Pública [SEP], 2007, 2009) y de que, a nivel gubernamental, existe la voluntad de analizar, diagnosticar y proponer directrices generales (Instituto Nacional de Evaluación Educativa [INEE], 2017) para planear un sistema educativo que garantice a la población indígena condiciones de inclusión y equidad, la meta de lograr un sistema eficiente e inclusivo para esta población está lejos de poderse alcanzar.

Partamos de los diagnósticos que han señalado las múltiples carencias que caracterizan al sistema de educación indígena aún en el presente siglo. Uno de los aspectos más relevantes es que la lengua de enseñanza en las aulas indígenas es el español, no la lengua originaria, a la cual tampoco se le considera como lengua de comunicación social en el ámbito escolar. Por su parte, la mitad de los docentes en preescolar y educación primaria no suelen ser hablantes de lengua indígena y, además, poseen menores niveles de preparación pedagógica que la media nacional (INEE, 2017). Resalta, asimismo, la inexistencia de metodologías claras que les ofrezcan a los docentes pautas realistas y coherentes con un modelo funcional en torno a la enseñanza de las lenguas en su contexto particular (Bertely, 2015).

A partir de lo anterior, el Instituto Nacional de Evaluación Educativa (INEE) delineó algunas directrices para resolver estos factores que determinan el fracaso de la educación intercultural; entre ellas destaca "la definición de metodologías y materiales que faciliten un bilingüismo equilibrado (dominio oral y escrito del español y de la lengua originaria propia) y que respondan a las necesidades, las expectativas y las características poblacionales" (2017, p. 7). De esta manera, la definición de una metodología de enseñanza clara e inclusiva se revela como un factor determinante para disminuir las carencias mencionadas en este sector de la población. ${ }^{1}$

Ante este panorama y la inexistencia de una solución estructural que, de presentarse, puede llegar a tardar mucho tiempo en dar resultados positivos, se han concretado algunas iniciativas locales que han hecho propuestas exitosas que han

1 Debemos señalar que estamos conscientes de que, en la enseñanza del español como lengua materna, la metodología de enseñanza tampoco está libre de controversia; pero esto no es razón para no abordar el problema en la educación intercultural. 
conseguido mostrar que es posible equilibrar en el aula y la comunidad escolar la relación diglósica y sustractiva entre las lenguas (Feltes, 2017; Hamel et al., 2016). En general, estos proyectos promueven una verdadera orientación intercultural bilingüe e incluso crítica, como sustituto de la castellanización que fue el modelo predominante en el siglo pasado, y su objetivo es lograr la preservación lingüística y afirmación cultural a través del aprendizaje de ambas lenguas. Ponemos mencionar la propuesta de método inductivo intercultural (Bertely, 2015), centrada en la formación docente, pero no solo como maestros de lengua originaria, sino como constructores de una cultura originaria que promueva el Buen Vivir, en donde "la ayuda y la reciprocidad se viven como prácticas políticas, sociales y productivas destinadas al bien común" (Bertely, 2015, p. 82).

La doble inmersión es el principio que otro proyecto aditivo implementado en la región Amuzga de Guerrero (zona centro-sur del país), y tal como lo anuncia, en esta metodología se distribuye el uso extensivo de ambas lenguas, en espacios diferentes, por lo que promueve la enseñanza equilibrada de temas curriculares, así como la enseñanza de la lectura y la comprensión lectora (Reese y Feltes, 2014).

Otra propuesta impactó desde inicios de este milenio a escuelas p'urhepechas de la Meseta Tarasca (Hamel et al., 2004, 2016), la cual está construida con bases teóricas y metodológicas de la sociolingüística y psicolingüística y se basa en el principio de transferencia de competencias, incluidas las de lectura y escritura, entre L1, la lengua originaria, y L2 para consolidar así una "proficiencia común subyacente" (Hamel et al., 2004). Es decir, según los investigadores, al afianzar las concepciones cognitivas propias de la comunidad en la lengua originaria se fortalecen los aprendizajes también en español (Hamel et al., 2004, 2016).

Creemos que estos y otros proyectos exitosos en un entorno restringido (Feltes, 2017) pueden fortalecerse con un enfoque sociocultural discursivo que provea una definición clara del lenguaje como una práctica social y postule el género discursivo como punto de referencia de planeación curricular, ya que este modelo entiende sistemáticamente la relación del lenguaje con los modelos culturales, las ideologías, los valores, las actitudes y los problemas interculturales de las comunidades y de las instituciones escolares.

\section{Hacia una metodología integral para la enseñanza de la lengua indígena}

\section{Los nuevos estudios de literacidad}

En los últimos años, uno de los intereses en el contexto de la enseñanza de la lengua es resaltar su naturaleza de actividad o práctica social situada y sus vínculos con la agencialidad en los contextos multiculturales (Zavala, 2011). En esta línea conceptual, se retoman propuestas como la teoría de las prácticas sociales (Reckwitz, 2002), para mostrar que el lenguaje y su aprendizaje son prácticas sociales que adquieren sentido en el interior mismo de las comunidades que las generan.

La teoría de las prácticas sociales reconoce que una práctica es un tipo de comportamiento reiterado que se compone de varios elementos relacionados, como movimientos corporales, formas de actividades mentales, objetos o instrumentos y la manera de utilizarlos, marcos de conocimiento y las formas de entenderlos; es decir, conocimientos sobre la forma de entender, saber hacer y comportarse que incluye también el nivel emocional. En pocas palabras, podemos decir que una práctica social es una rutina, una forma convencionalizada en la que movemos el cuerpo, manipulamos los objetos, describimos las cosas, entendemos el mundo y hablamos de él. Por lo mismo, al aprender una práctica vamos modelando nuestra mente dando forma a modelos o patrones colectivos. El conocimiento de la práctica, por ejemplo las actividades comunitarias o las actividades escolares, incluye formas de entendimiento, de saber hacer, de desear y sentir que están íntegramente conectadas entre sí. En este marco de referencia, las prácticas del lenguaje, como prácticas sociales, son estructuralmente semejantes a cualquier otra práctica; son también patrones corporales (formas de gesticular), formas de comprender y saber hacer (conocimientos pragmáticos y gramaticales), 
objetos (modos y soportes para la comunicación) e intenciones (propósitos comunicativos y sociales).

Por su parte, el concepto de agencialidad (Bourdieu, 1977; Giddens, 1995, Reckwitz, 2002) se refiere a la capacidad de los individuos de modificar o transformar las estructuras sociales a fin de configurar sus roles en las prácticas socioculturales en las que participan. Es decir, la agencialidad humana se da en la realización de las prácticas; esta se ejerce y expresa en y a través de la ejecución de una práctica, $y$ en realizarla de tal forma que sea reconocida por otros. Así, la agencialidad conecta directamente a la teoría de la práctica social con la noción de Discurso (con $D$ mayúscula) y el problema de la identidad (Gee, 2001).

En esta perspectiva, se entiende por Discurso las maneras de combinar y coordinar palabras, hechos, pensamientos, valores, cuerpos, objetos, herramientas, tecnologías y otras personas, en situaciones específicas, para representar y reproducir identidades y actividades específicas socialmente situadas (Gee, 2001). Las identidades son las formas como somos reconocidos por otros como un determinado tipo de persona en un contexto dado; así, se pone el énfasis en los tipos de prácticas involucradas en el mantenimiento de nuestras identidades. Se parte del supuesto de que el aprendizaje de una práctica (lectura, escritura u oralidad), solo es eficaz si lo que se aprende está conectado de manera significativa con el contexto en el que se sitúan esas prácticas y el reforzamiento que hace de nuestras identidades. En este entendido, el aprender una lengua es aprender a ser y hacer con el lenguaje para ser reconocidos como miembros de las distintas comunidades de práctica y comunidades discursivas en las que interactuamos. Se trata de un aprendizaje que atraviesa los espacios culturales para sumar el aprendizaje de la lengua y la cultura de la comunidad y el hogar a las destrezas académicas aprendidas en la escuela (Barton y Hamilton, 1998; Gee, 2004).

Retomando este planteamiento, los nuevos estudios de la literacidad sostienen que para entender la lectura y la escritura no basta con conocer los procesos cognitivos llevados a cabo por los sujetos, sino que además se debe comprender lo que estos hacen cuando leen y escriben y para qué lo hacen. En esta línea, la literacidad se entiende como un proceso que, además de involucrar la adquisición de habilidades cognitivas, implica un conjunto de prácticas sociales que pueden ser inferidas a partir de los eventos mediados por textos escritos (Barton y Hamilton, 1998). En consecuencia, se enfatiza la importancia de las prácticas existentes en las relaciones personales dentro de comunidades o grupos, $\mathrm{y}$ de los eventos, es decir, las actividades y tareas que se observan cuando las personas leen y escriben. De esta manera, las prácticas se determinan a partir de las normas sociales en las que las personas leen y escriben, y, además, regulan el uso, la distribución, la producción y el acceso a los textos; mientras que, en los eventos, se observan los episodios que se construyen en las prácticas de literacidad.

En una determinada comunidad, la escritura, la lectura y las interacciones orales tienen propósitos claros y objetivos sociales más amplios. Esto es lo que nos permite diferenciar, por ejemplo, las prácticas de literacidad escolar de las prácticas vernáculas, por citar solo dos casos. En ambas literacidades están en juego ideologías, relaciones de poder y discursos que regulan el uso y la distribución de textos, legitiman cierto tipo de conocimientos y posibilitan su producción y su acceso.

En el contexto educativo, la lectura, la escritura y la oralidad se convierten en prácticas sofisticadas y con un mayor valor que aquellas otras prácticas propias de contextos no institucionalizados. En contraste, en las instituciones menos dominantes, como asociaciones, grupos deportivos, grupos de tarea, grupos vecinales o comunitarios, las prácticas suelen no estar sistematizadas o reguladas institucionalmente. Por lo mismo, el conocimiento que se genera no suele estar valorado como un aprendizaje significativo, puesto que se aprende de manera informal en espacios como los hogares, las comunidades, las familias o cualquier situación cotidiana. A este tipo de prácticas se les conoce como prácticas de literacidad vernáculas (Hamilton, 2000). Al adquirirse de manera informal, integran lenguajes de 
otros ámbitos o dominios. Son prácticas híbridas en las que los usos, reglas, estrategias y propósitos son determinados por un conocimiento local. Se trata de prácticas diferentes, pero no por ello de menor importancia que las prácticas escolares.

Para que el aprendizaje de la lengua sea eficaz, es necesario que esté conectado de manera significativa y motivacional con las versiones reconocidas por los miembros de la comunidad. En este sentido, coincidimos con los autores que afirman que para que el aprendizaje sea eficaz es necesario pensar la educación y el aprendizaje en términos de pensar las vidas humanas como trayectorias a través de las distintas prácticas sociales, escolares y vernáculas (Gee et al., 1996), pues aprender los usos sociales del lenguaje significa progresar hacia una comprensión del ser y hacer, de manera tal que el estudiante se coloque en el camino correcto para convertirse en un participante competente de la vida real, cualquiera que sea el Discurso o contexto en el que se encuentre.

$\mathrm{Al}$ entender, por lo tanto, la vida de una cultura como un conjunto complejo en términos de potencial de acción y significado (Bazerman, 2012: Halliday, 1994), el género discursivo se convierte en una referencia para entender la vida sociosemiótica de las culturas; ${ }^{2}$ por ello, este concepto nos provee el soporte teórico-metodológico para convertir las prácticas sociales en objetos de enseñanza. La experiencia australiana, como lo veremos más adelante, nos demuestra que una propuesta de educación que promueva una relación aditiva y crítica entre lenguas que conviven en un contexto parecido al mexicano (Rose, 1999) puede hallar en una metodología de este tipo una alternativa que relaciona los aspectos ideológicos, las cosmogonías culturales de cada comunidad discursiva, con los recursos semánticos y lexicogramaticales que instancian los significados en los textos que todas las lenguas crean.

2 Tomamos en este trabajo la definición de Rose y Martin $(2012,2018)$ del género como la realización de un propósito social llevado a cabo a través de una secuencia de etapas funcionales.

\section{La pedagogía del género discursivo como forma de vivir el enfoque sociocultural en las aulas}

Con antecedentes de desventaja y exclusión de la población indígena australiana muy semejante a la descrita en México (Rose, 2004), en la década de los ochenta, en ese país se inició el desarrollo de los programas basados en el género discursivo con el fin de cambiar las prácticas vigentes en ese momento, basadas en metodologías anglosajonas como el lenguaje total (whole language, Goodman, 1986 citado en Rose y Martin, 2018) que promueven "el descubrimiento" individual en el aprendizaje de la lectura y escritura. Estos métodos, según Rose y Martin (2018), lejos de favorecer a grupos catalogados como con dificultades de aprendizaje, consolidan la desigualdad de aprendizaje en las aulas, pues favorecen el contexto de niños pertenecientes a una clase media, cuyo contexto de crianza les provee una formación consistente en cultura escrita (Rose, 2015), y no valoran las prácticas orales que son fundamentales en la vida de las comunidades indígenas, por ejemplo.

Por lo tanto, basados en el principio de andamiaje vigotskiano, se emprendió una serie de proyectos pedagógicos cuyas prácticas pedagógicas promueven el acompañamiento continuo e intensivo de los docentes hacia los estudiantes; las situaciones de enseñanza se convirtieron, así, en exploraciones conjuntas del potencial de significación de los textos, uno de los principios más fuertes de la metodología que se estaba configurando (Rose et al., 1999). Es dentro de estos programas incipientes donde Rose ubica el origen del concepto de género de la Escuela de Sydney (Rose 1999; Rose y Martin, 2012, 2018), el cual se consolidó dentro de la teoría sistémico-funcional y constituye la base teórica de lo que, a principios del nuevo siglo, se conoció como el programa Reading to Learn ${ }^{3}$ (R2L, en adelante) (Rose, 2015).

Tal como lo describe Rose (2006), R2L integra a su propuesta pedagógica una teoría sociológica del

3 Al inicio, el nombre completo del programa fue Learning to read: reading to learn (Rose, 1999; Rose et al., 2003). Posteriormente, se abrevió a Reading to learn (Rose y Martin, 2012 y 2018). 
mundo del lenguaje escolar basado en Bernstein (1990), que promueve una mayor equidad y responsabilidad social (Christie y Simpson, 2010; Rose, 1999), pues propone que la tarea pedagógica debe plantearse desde una pedagogía "visible" (Bernstein, 1990), en donde los criterios, las normas y las secuencias de planeación y evaluación se hagan explícitos. Asimismo, sugiere abandonar la práctica tan difundida del IRF o diálogo triádico (Cazden, 2001) durante las intervenciones en el aula, pues se busca que el lenguaje del maestro empodere la participación de los estudiantes más débiles (Rose y Martin, 2012, 2018) en lugar de privilegiar la intervención de quienes son más aventajados, como se ha demostrado que el IRF lo hace. Además, su ciclo pedagógico (Hernández Ramírez, 2010; Martin, 1999, 2012; Rose y Martin, 2012, 2018) permite al docente planear en torno a un género discursivo actividades compartidas de comprensión y producción de textos completos que encarnan las prácticas de literacidad de la cultura, e incluso, compararlas con las de otra cultura sin detrimento de ninguna de ellas, sino entendiendo su especificidad y apoyándose mutuamente.

Este ciclo organiza las actividades en una secuencia de mayor acompañamiento o andamiaje hasta etapas de paulatina independencia, todas ellas dirigidas por el docente, quien debe constituirse en un experto capacitado para mostrar cómo el lenguaje significa en sus diversos niveles (discursivo, semántico y lexicogramatical, incluyendo los recursos multimodales), y cómo estos se convierten en estrategias que los autores proponen para lograr una determinada acción social. La independencia en el ejercicio de una voz discursiva consciente, autónoma y empoderada es el paso siguiente del proceso educativo, metas que apoyan el enfoque sociocultural que hemos enfatizado en este trabajo.

En resumen, se trata de un enfoque que instrumenta un programa de enseñanza de la literacidad a través de acciones de acompañamiento y colaboración, que dan a los estudiantes en desventaja herramientas para actuar en el mundo real (Martin, 1999), las cuales, como Martin lo ha enfatizado, les han sido negadas - en el mejor de los casos por omisión-a los sectores marginados de cualquier sociedad. Además, la metodología provee a los docentes de un sólido marco teórico de descripción lingüística sobre los sistemas que componen la arquitectura del lenguaje (Halliday, 1994), una metodología de análisis del discurso y una amplia gama de descripciones de géneros y subgéneros escolares en diversas áreas disciplinares (Christie y Derewianka, 2008; Martin y Rose, 2008; Moyano, 2013).

El R2L ha sido difundido en diversas escuelas indígenas y no indígenas en Australia y otras regiones del mundo en los diferentes niveles escolares: primaria, secundaria (Board of Studies Nsw, 2006) y superior (Rose et al., 2003, 2008), incluso, en enseñanza de matemáticas (Board of Studies Nsw, 2008), con resultados muy provechosos, pues se logra que los estudiantes indígenas desarrollen sus prácticas de literacidad, tal como se espera en los programas oficiales (Rose, 2015). Asimismo, en Iberoamérica ha sido difundido y ejercido con éxito en diversos niveles de escolaridad en España (García Parejo y Whittaker, 2017), en Chile (Westhoff, 2017), y en Colombia (Rojas et al., 2016).

En México, esta metodología guía las pautas pedagógicas de los programas de literacidad académica en la Facultad de Filosofía y Letras de la UATX (Hernández, García y Rosales, 2014; Hernández Ramírez, 2019) y, recientemente, siguiendo esta línea, en la misma institución se trabajan proyectos de literacidad orientados a promover propuestas para la enseñanza del náhuatl como lengua indígena y a lograr descripciones funcionales de esta lengua, tales como el proyecto Leer para aprender con poesía contemporánea en náhuatl (Hernández y Delgado Aguilar, 2020) y Procesos de literacidad en la educación multicultural bilingüe en el estado de Tlaxcala (Castro et al., 2020).

Así pues, tomando en cuenta todos estos aspectos, a continuación describiremos brevemente algunas pautas del proyecto de intervención sobre el género recuento en náhuatl (Rose y Martin 2012, 2018) que se halla en fase de planeación en nuestra universidad. 


\section{El recuento en náhuatl: un ejemplo de intervención integral}

La narración de acontecimientos es un modo de organización del discurso cuyos rasgos lo distinguen claramente de otras formas de organización, como la descripción o la exposición, y cuyo uso permite que los hablantes produzcan narraciones en prácticamente todas las situaciones de comunicación. Por lo mismo, muchos investigadores han planteado que esta estructura textual debería considerarse básica en la educación. De hecho, hay quienes sostienen que la narrativa es una forma primaria de discurso que da origen a otras formas discursivas y que es la primera que se aprende (Beals y Snow, 1994; Bruner, 1990). Autores como Nelson (1986) y Eisenberg (1985) han mostrado que las primeras producciones narrativas de los niños corresponden a eventos de la vida cotidiana que suceden más de una vez, como por ejemplo bañarse, ir a la escuela o celebrar un cumpleaños. Desde una teoría del género, el "contar" se constituye como una familia de géneros discursivos (Ciapuscio, 2007) fundamentales en las prácticas vernáculas y escolares (Rose y Martin, 2018) y que forman parte del currículo escolar desde el preescolar.

Ahora bien, un punto central en el estudio de la narración infantil es su papel en el proceso de alfabetización inicial; por lo mismo, hay un enorme interés por identificar los géneros narrativos en la primera infancia y su orden de aparición en el desarrollo del lenguaje infantil. Al respecto, una de las perspectivas más influyentes es aquella que distingue la aparición de los géneros a partir de las interacciones en las que se realizan las narraciones. Algunos autores han identificado como los géneros iniciales el recuento o el relato, el cuento o la anécdota (Christie y Derewianka, 2008; Heath, 1986; Hicks, 1988). En este trabajo nos centramos, particularmente, en el primero de ellos.

El recuento es una narración cotidiana cuyo objetivo es responder a la pregunta ¿qué sucedió? Básicamente, se trata de una narración casual en la que el niño puede recordar y poner por escrito sucesos (fui al cine, llovió muy fuerte, le dolió la cabeza, etc.), y ordenarlos en secuencias temporales muy cercanas (ayer, hoy, el fin de semana) sin la presencia de una complicación o problema que resolver en el relato. La producción de recuentos familiariza al niño con estructuras narrativas simples que sirven para dar a conocer a otros, situaciones reales relativas a su propia experiencia o la ajena. Después, estas construcciones relativamente simples dan paso a géneros mucho más complejos, como el cuento o la historia, que son narraciones con complicación y propósitos de tipo estético o literario.

Como hemos enfatizado con anterioridad, para los niños que inician su formación escolar en contextos bilingües, el reto del aprendizaje de la lectura y la escritura supone un esfuerzo mucho mayor. Al aprendizaje del código de representación escrita de la lengua originaria y/o de la lengua de instrucción, hay que sumar aspectos como la construcción del sujeto como autor, la descolonización del conocimiento local y su valoración positiva, explorar los compromisos de las comunidades poseedoras del saber tradicional que se pretende escribir, entre muchas otras cosas (Nava, 2019).

En la cultura oral nahua se puede ver el recuento como uno de los géneros que materializan las prácticas sociales de las comunidades, pues su vida social cotidiana es rica en narraciones y constituyen un vehículo importante de los valores vigentes en su cosmogonía. En los relatos, se visibiliza la postura del hablante ante los hechos vividos y se ofrece una versión crítica a partir de los valores locales. Por ello, creemos que un proyecto de bilingüismo aditivo basado en una pedagogía del género discursivo enseñará a contar o narrar haciendo consciente al niño de las diversas formas que esta acción social puede revestir en las culturas que conviven en su contexto, sumando sistemáticamente a su potencial de significación y producción discursiva esta variedad. Asimismo, este aprenderá a explicar, a defender las posturas propias y colectivas, e incluso a elaborar pensamiento matemático en cualesquiera que sean las lenguas de enseñanza.

Antes de concluir, se ofrece una breve exposición de cuatro principios que consideramos el 
fundamento de estos programas en la etapa de preparación para la escritura del recuento, en principio, $\mathrm{y}$ de cualquier género en lengua náhuatl.

\section{Posicionamiento identitario como miembro activo de una comunidad}

En una comunidad, todos contribuyen a la construcción de la cognición social. En este sentido, los seres humanos sienten el imperativo de comunicar sus experiencias a los demás. Esta práctica los valida como miembros activos de ese colectivo. Son precisamente estas formas de participación las que le dan sentido a un grupo social, sea este una familia, una colonia o un grupo escolar. Cada integrante está orientado a la promoción del crecimiento de su comunidad y para ello debe asumirse como agente enculturador ya que esa persona puede ser la única que ha vivido dicha experiencia y, por lo tanto, su aportación será referente para los demás. De esta manera, los estudiantes tomarán mayor conciencia de su papel agentivo en el mantenimiento y crecimiento de la comunidad en la que ellos son los actores principales: su salón de clases.

\section{Reflexión sobre los valores socioculturales locales}

En la producción de un recuento están implicados varios procesos discursivos. Uno de ellos es la evaluación que hacemos de la experiencia a partir de la matriz cultural del grupo. Por lo tanto, el profesor podría guiar la reflexión entre sus estudiantes acerca de algunos aspectos de la experiencia que se va a contar. Esta reflexión les permitirá reafirmar sus valores socioculturales, tomar postura y presentarla en su texto, siguiendo así la estructura retórica del recuento en su lengua.

\section{Valoración personal de la experiencia}

Los recuentos, en cuanto experiencias discursivizadas, requieren de la valoración personal del hablante. Al tratarse de una experiencia personal, el sujeto hace visibles su emoción o actitud ante lo vivido. En este caso, el profesor, junto con los estudiantes, puede explorar las diversas maneras en que dentro de la cultura y, particularmente, en el recuento se expresa la valoración.

\section{Selección de los recursos léxico-gramaticales}

Los hablantes nos enfrentamos a la selección de aquellos recursos léxico-gramaticales que consideramos idóneos para construir la significación buscada. Estos recursos dependen de varios factores, entre los que se destaca la identidad sociocultural del interlocutor. En náhuatl, por ejemplo, existen tres niveles de habla para referirse a una persona; la selección del nivel adecuado se da en función del grado de cercanía social o relación ritual entre los participantes. Por ello, algunos aspectos de la lengua como sistema formal deben ser trabajados en clase, pues el discurso de cada género implica diferencias léxico-gramaticales muy importantes.

Como se puede observar a partir de lo expuesto, el trabajo de los géneros vernáculos como el recuento ofrece oportunidades de aprendizaje que la enseñanza tradicional hace a un lado. De manera resumida señalaremos que se consolidan aspectos de la cultura local, se entrena al estudiante para tomar postura, valorar hechos y construir perspectiva, y se promueven competencias en el uso del lenguaje que le son útiles al alumno para su desempeño inmediato no solo en el aula, sino en la vida cotidiana. De igual forma, el trabajo con los géneros, vernáculos o escolares, ofrece la oportunidad de ir construyendo acervos de textos escritos en la lengua originaria, hoy casi inexistentes, que sirvan de apoyo en el aula para la promoción de una alfabetización bilingüe que realmente permita a los niños de nuestras comunidades indígenas valorar su lengua materna como medio de comunicación, y como objeto y fuente de conocimiento, para hacer realidad lo planteado por la nueva escuela mexicana. 


\section{Conclusiones}

Las limitaciones en la enseñanza de la lectura, la escritura y la oralidad de las lenguas originarias en nuestro país no serán superadas a corto plazo si no se integran a la investigación y al trabajo en el aula enfoques integradores orientados a mejorar el desarrollo de los conocimientos necesarios para el aprendizaje de los discursos ( $\mathrm{y}$ sus géneros), y de las identidades en los dominios de interacción multi- e intercultural. Estamos convencidos de que, desde los estudios socioculturales, los nuevos estudios de la literacidad y la pedagogía del género se pueden proponer teorías y descripciones de esas prácticas que pueden ser útiles en las condiciones de la escuela en las comunidades indígenas del país.

La enseñanza de la lectura y la escritura a partir del reconocimiento y la producción de géneros discursivos como el recuento aprovecha el interés y la curiosidad propias de la infancia y el conocimiento social para acercarlos progresivamente a las prácticas de lectura y escritura, pues al enseñar a leer, a comprender, a construir significado y a escribir con un fuerte andamiaje y estableciendo comparaciones entre los diversos ámbitos de construcción semiótica, estamos favoreciendo el aprendizaje del uso del lenguaje como herramienta de comunicación. Este aprendizaje e interacción regulan el pensamiento intercultural y orientan la conducta propia y ajena de manera comprensiva y aditiva, no restrictiva. Es decir, estamos ofreciendo a los niños indígenas, afrodescendientes y todos aquellos en situación vulnerable, la posibilidad de adquirir y comunicar experiencias en torno a la lectura, la escritura y la oralidad como algo útil en los diversos ámbitos, no solo de la vida escolar, también de la personal y social. Por lo mismo, la escuela tiene la función de enseñar los conocimientos relevantes de la cultura en la que el niño está inmerso, es decir, los géneros que son importantes para la construcción de su propia historia como miembro de su comunidad. Al ser la encargada y responsable de la enseñanza de la lectura y la escritura, la escuela no puede dejar a un lado los conocimientos y experiencias infantiles aportados por su tradición cultural, sino que debe partir de ellos para acercarlo de forma paulatina al saber formal exigido por su comunidad y por los entornos profesionales y laborales a los que todos los ciudadanos tienen derecho a incorporarse para lograr la tan necesaria equidad.

\section{Referencias}

Barriga, R. (2018). De Babel a Pentecostés: Políticas lingüisticas y lengua indígena, entre historias, paradojas y testimonios. Secretaría de Educación Pública/ Coordinación General de Educación Intercultural Bilingüe.

Barton, D. y Hamilton, M. (1998). Local literacies: Reading and writing in one community. Routledge.

Bazerman, C. (2012). Géneros textuales, tipificación y actividad. BUAP.

Beals, D. y Snow, C. (1994). Thunder is when the angels are upstairs bowling: Narratives and explanations at the dinner table. Journal of Narrative and Life History, 4(4), 331-352.

Bernstein, B. (1990). La construcción social del discurso pedagógico. Mario Díaz Editor.

Bertely, M. (2015). Enfoques postcoloniales y movimiento político y pedagógico intercultural en y desde Chiapas, México. Relaciones: Estudios de Historia y Sociedad, 36(141), 75-102. http://www. scielo.org.mx/scielo.php?script=sci_arttext\&pi$\mathrm{d}=$ S0185-39292015000100075\&lng=en\&nrm =iso\&tlng=es

Board of Studies Nsw. (2006). Scaffolding the English curriculum for Aboriginal secondary students NSW 7-10 English Syllabus. Aboriginal Support Pilot Project. Office of the Board of Studies.

Board of Studies Nsw. (2008). The journey's just begun: Enhancing schools' capacity to partner Aboriginal communities to improve student learning. Prepared on the Crown in right of the State of New South Wales.

Bourdieu, P. (1977). Outline of a theory of practice. Cambridge University Press.

Bruner, J. (1990). Acts of meaning. Harvard University Press.

Cassany, D. (2008). Prácticas letradas contemporáneas. Ríos de Tinta. 
Castro, M. C., Hernández, L. A. y Nava, R. (2020). Proyecto: Procesos de literacidad en la educación multicultural bilingüe en el estado de Tlaxcala, México. Facultad de Filosofía y Letras. Universidad Autónoma de Tlaxcala.

Cazden, C. B. (2001). Classroom discourse: The language of teaching and learning. Heinemann.

Christie, F. y Derewianka, B. (2008). School discourse. Continuum.

Christie, F. y Simpson, A. (Eds.) (2010). Literacy and social responsibility: Multiple perspectives. Equinox.

Ciapuscio, G. (2007). Genres et familles de genres: apports pour l'acquisition de la compétence générique dans le domaine académique. Études de Linguistique Apliquée, 148, 405-416.

Delgado Aguilar, J. (2019). Leer para aprender con poesía contemporánea en náhuatl. [Tesis de maestría, Universidad Autónoma de Tlaxcala].

Diario Oficial de la Federación (DOF). (2019). Ley general de educación.

Dietz, G. y Mateos, L. (2011). Interculturalidad y educación intercultural en México: Un análisis de los discursos nacionales e internacionales en su impacto en los modelos educativos mexicanos. Secretaría de Educación Pública/Coordinación General de Educación Intercultural Bilingüe.

Eisenberg, A. (1985). Learning to describe past experiences in conversation. Discourse Process, 8, 177-204.

Feltes J. M. (2017). Metodologías pedagógicas para el desarrollo de las habilidades del bilingüismo, la biliteracidad y la comprensión intercultural en dos o más lenguas nacionales. INEE.

García Parejo, I. y Whittaker, R. (2017). Presentación: Teoría y práctica del modelo Reading to Learn (Leer para aprender) en contextos educativos transnacionales. Lenguaje y textos, 46, 1-5. http:// doi.org/10.4995/lyt.2017.8722

Gee, J. P., Hull, G. y Lankshear, C. (1996). The new work order: Behind the language of the new capitalism. Westview Press.

Gee, J. P. (2001). Reading as situated language: A sociocognitive perspective. Journal of Adolescent and Adult Literacy, 44(8), 714-725.

Gee, J. P. (2004). Identity as an analytic lens for research in education. Review of Research in Education, 25, 99-125.
Gee, J. P. (2006). A sociocultural perspective on early literacy development. En S. B. Neuman y D. K. Dickinson (eds.), Handbook of early literacy research (pp. 30-42). Guilford Press.

Giddens, A. (1995). La construcción de la sociedad: Bases para una teoría de la estructuración. Amorrortu.

Halliday, M. A. K. (1994). Introduction to functional grammar. Edward Arnold.

Hamel, R. E.; Brumm, M.; Carrillo Avelar, A.; Loncon, E.; Nieto, R. y Silva Castellón, E. (2004). ¿Qué hacemos con la Castilla?: La enseñanza del español como segunda lengua en un currículo intercultural bilingüe de educación indígena. Revista Mexicana de Investigación Educativa, 9(20), 83-107.

Hamel, R. E., Erape, A. E., Hernández Burg, M. y Márquez E., H. B. (2016). T’arhexperakua Creciendo juntos: Investigación-acción colaborativa entre maestros p'urhepechas, investigadores y estudiantes de la UAM para el desarrollo de una educación intercultural bilingüe. Bricolage. https:// revistabricolage.wordpress.com/2016/06/20/ tarhexperakua-creciendo-juntos/

Hamilton, M. (2000). Expanding the new literacy studies. En D. Barton, M. Hamilton y R. Ivanic (eds.). Situated literacies: Reading and writing in context (pp. 15-32). Routledge.

Heath, S. B. (1986). Taking a cross-cultural look at narrative. Topic in Language Disorders, 7(1), 84-94.

Heller, M. (2007). Bilingualism as ideology and practice. En M. Miller (ed.), Bilingualism: A Social Approach (pp. 1-22). Palgrave.

Hernández Ramírez, L. A. (2010). La pedagogía basada en el género de la lingüística sistémico funcional: Aprender la lengua, aprender con la lengua y aprender sobre la lengua. Lingüística Mexicana, 5(1), 67-89.

Hernández Ramírez, L. A. (2019). La definición en el registro académico: Pautas para su intervención en el aula. En M. C. Castro Azuara, (coord.), Literacidad académica y disciplinar: Retos y propuestas de intervención en el nivel superior (pp. 99-125). Clave Editorial, AM Editores, y UATX.

Hernández Ramírez, L. A., García Torres, O. y Rosales Rodríguez, A. (2014). Literacidad en la educación superior: Gramática del español, un curso de inserción básica en el mundo de la literacidad ciudadana. En L. A. Hernández Ramírez, (coord.), La enseñanza de lenguas en discusión: Proyectos y experiencias de 
gestión en lenguas extranjeras, literacidad académica y digital y lenguas originarias (pp. 227-238). Universidad Autónoma de Tlaxcala.

Hernández, L. A. y Delgado Aguilar, J. (2021). Enseñar náhuatl a través de la poesía: Una intervención didáctica desde la pedagogía de género; leer para aprender. Íkala, 26(1), 139-163. https://doi. org/10.17533/udea.ikala.v26n01a09

Hicks, D. (1988). The development of genre skills: A linguistic analysis of primary school children's stories; Report and eventcast [tesis inédita de doctorado]. Harvard Graduate Schools of Education.

Instituto Nacional de Evaluación Educativa (INEE). (2017). Directrices para mejorar la atención educativa de niñas, niños y adolescentes indígenas. INEE.

Martin, J. R. (1999). Mentoring semogénesis: 'genre-based' literacy pedagogy. En F. Christie (ed.), Pedagogy and the shaping of consciousness: Linguistic and social processes (pp. 123-155). Cassel.

Martin, J. R. y Rose, D. (2008). Genre relations: Mapping culture. Equinox.

Moyano, E. (Coord.). (2013). Aprender ciencias y humanidades: Una cuestión de lectura y escritura; Aportes para la construcción de un programa de inclusión social a través de la educación lingüística. Universidad Nacional de General Sarmiento.

Muñoz, E. (2017). Interculturalizaciones: Transiciones, mediaciones y conflictos en lenguas, comunidades y educación escolar. UAM.

Nava, R. (2019). Procesos de literacidad en lengua originaria: Desarrollo de competencias para la escritura académica en náhuatl. En M. C. Castro Azuara (coord.), Literacidad académica y disciplinar: Retos $y$ propuestas de intervención en el nivel superior. AM Editores/Universidad Autónoma de Tlaxcala, 171-191

Nelson, K. (Ed.) (1986). Event knowledge: Structure and function in development. Lawrence Erlbaum Associates.

Organización de las Naciones Unidas para la Educación, la Ciencia y la Cultura (Unesco). (2006). Directrices de la Unesco sobre la educación intercultural.

Reckwitz, A. (2002). Toward a theory of social practices: A development in social theorizing. European Journal of Social Theory, 5(2), 243-263.

Reese, L. y Feltes, J. M. (2014). La implementación de programas de doble inmersión en escuelas multigra- dos rurales indígenas. Sinéctica, Revista Electrónica de Educación, 43, Iteso. http://www.sinectica.iteso. $\mathrm{mx} /$ articulo/?id=43_la_implementacion_de_programas_de_doble_inmersion_en_escuelas_multigrados_rurales_indigenas

Rogers, R. (2002). Between contexts: A critical analysis of family literacy, discursive practices, and literate subjectivities. Reading Research Quarterly, 37, 248-277. https://www.researchgate.net/publication/233896004_Between_contexts_A_critical_analysis_of_family_literacy_discursive_practices_and_literate_subjectivities

Rogoff, B. y Gutiérrez, K. (2010). Maneras culturales de aprender: Rasgos o repertorios de práctica. En S. Frisancho, M. T. Moreno, P. Ruiz Bravo y V. Zavala (eds.), Aprendizaje, cultura y desarrollo: Una aproximación interdisciplinaria (pp. 19-25). Pontificia Universidad Católica de Perú.

Rojas, I., Olave Arias, G. y Cisneros Estupinán, M. (2016). Alfabetización académica y pedagogía de género discursivo en la lingüística sistémico-funcional: Una experiencia de trabajo. Rev. Signos, 49(1), 224-246. http://dx.doi.org/10.4067/ S0718-09342016000400011

Rose, D. (1999). Culture, competence and schooling: Approaches to literacy teaching in indigenous school education. En F. Christie (ed.), Pedagogy and the shaping of consciousness: Linguistic and social processes (pp. 217-245). Cassel.

Rose, D. (2004). Sequencing and pacing of the hidden curriculum: How indigenous learners are left out of the chain. En J. Muller, A. Morais y B. Davies (eds.), Reading Bernstein, Researching Bernstein (pp. 91-107). Routledge Falmer.

Rose, D. (2006). Literacy and equality in the classroom. En A. Simpson, Proceedings of the National Conference on Future Directions in Literacy: Future Directions in Literacy Conference. University of Sydney.

Rose, D. (2015). Teaching reading and writing with Aboriginal children. En N. Harrison, Teaching and learning in Aboriginal education (3. ${ }^{\mathrm{a}}$ ed.). Oxford University Press.

Rose, D., Gray, B. y Conwey, W. (1999). Scaffolding reading and writing for indigenous children in school. En P. Wignell (ed.), Double power: English literacy in indigenous schooling (pp. 30-66). Languages Australia. 
Universidad Pedagógica Nacional

Facultad de Humanidades

Rose, D., Lui-Chivizhe, L., McKnight, A. y Smith, A. (2003). Scaffolding academic reading and writing at the Koori Centre. Australian Journal of Indigenous Education [30th Anniversary Edition] (41-49). http://www.atsis.uq.edu.au/ajie/docs/2003324149. pdf

Rose, D. y Martin, J. R. (2012). Learning to write, reading to learn: Genre, Knowledge and Pedagogy in the Sydney School. Equinox.

Rose, D. y Martin, J. R. (2018). Leer para aprender: Lectura y escritura en las áreas del currículo. Editorial Pirámide.

Secretaría de Educación Pública. (SEP). (2007). El enfoque intercultural en educación. Orientaciones para maestros de primaria. Secretaría de Educación Pública/Coordinación General de Educación Intercultural Bilingüe.

Secretaría de Educación Pública. (SEP). (2009). Lineamientos generales para la educación intercultural bilingüe para las niñas y los niños indígenas. Secretaría de Educación Pública/Dirección General de Educación Indígena.

Wenger, E. (2001). Comunidades de práctica: Significado, aprendizaje e identidad. Paidós.

Westhoff, I. (2017). Desarrollo de una secuencia didáctica basada en el programa Leer para Aprender: Propuesta de aproximación al género narración en la educación primaria en Chile. Lenguaje y Textos, 46, 19-28. http://doi.org/10.4995/lyt.2017.8722

Zavala, V. (2011). La escritura académica y la agencialidad de los sujetos. Cuadernos Comillas, 1, 52-65. https://d1wqtxts1xzle7.cloudfront. net/32528247/5_zavala.pdf?1386749656=\&responsecontentdisposition=inline $\% 3 \mathrm{~B}+$ filename $\% 3 \mathrm{D}$ Virginia_Zavala_La_escritura_academica_y. pdf\&Expires $=1599765118 \&$ Signature $=$ ByM. 